\title{
EVEN AND ODD MODES ON A CONDUCTOR-BACKED SLOTLINE
}

\author{
Jan Zehentner, Jan Machac, Jan Mrkvica \\ Czech Technical University, Technicka 2, 16627 Prague 6, Czech Republic
}

\section{INTRODUCTION}

Printed-circuit lines have become the standard transmission lines in today's MIC. During the last thirty years hundreds of papers have discussed their properties, their behaviour and the modifications most suitable for particular applications. The conductor-backed coplanar waveguide and the conductor-backed slotline (CBSL) are interesting transmission lines since they enable circuits to be located on a metal base, ensuring improved mechanical strength, good heat sinking and easy dc biasing. Their great drawback is a leakage of power in the substrate resulting in undesired coupling to the neighbouring circuit sections. That is why the CBSL has stood somewhat at the margin of the interest of designers and only a few publications [1-3] have worked on analyzing it.

The purpose of this paper is to bring the attention of designers back to the CBSL and to clarify the line features in terms of the even and odd mode dispersion characteristics. Two new dominant modes are revealed and the influence of line dimensions on CBSL properties is presented. The dependence of the dispersion characteristics on the slotwidth exhibits a new unexpected evolution not previously observed on other planar transmission lines. Measurement of the even dominant wave and the even 1st leaky wave confirms theoretically derived observations. Conclusions drawn from the analysis result in recommendations for CBSL users.

\section{DISPERSION CHARACTERISTICS OF EVEN AND ODD MODES}

We analyzed the CBSL with the cross-section shown in Fig. 1, by the method of moments applied in the spectral domain. For this purpose we produced a code calculating the propagation constant of a selected mode. The second code calculates the electric field distribution of that mode on the cross-sectional plane by means of the backward Fourier transform. We distinguish even and odd modes on the CBSL

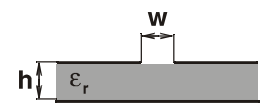

Fig. 1 Cross-section of a conductor-backed slotline. according to the transversal electric field distribution within the slot with regard to
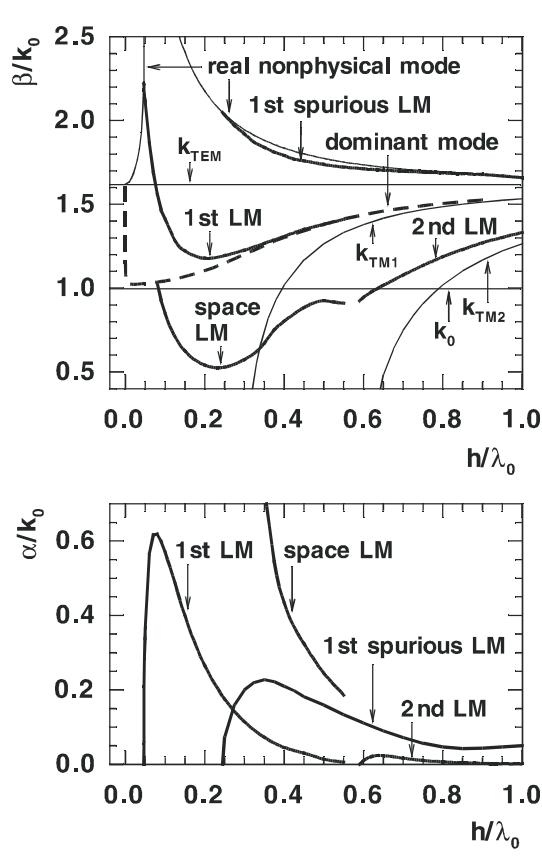

Fig. 2 Normalized dispersion characteristics of the even dominant and even leaky modes on the CBSL with $\mathrm{w}=6 \mathrm{~mm}, \mathrm{~h}=6 \mathrm{~mm}$ and $\varepsilon_{\mathrm{r}}=2.6$. the symmetry plane of the line. In addition, we will discuss the CBSL with a „narrow" slot, i. e., narrower than the substrate thickness and with a ,wide“" slot, i. e., wider than the substrate thickness.

On a transmission line consisting of three conductors and at least two dielectrics in the cross-section, as in the CBSL case, two dominant hybrid or quasi TEM waves can propagate when the frequency increases from zero. These waves have even and odd symmetry. The propagation constants of the even modes on the ,narrow" slotted CBSL, normalized to the propagation constant of free space $\mathrm{k}_{0}$, depending on the normalized frequency $\mathrm{h} / \lambda_{0}$, termed dispersion characteristics, are shown in Fig. 2. It is stated in [1] that the CBSL will always leak power into the dielectric-filled parallel-plate region. On the contrary, our analysis shows that the even dominant mode may propagate alone on the CBSL from dc up to the frequency at which the first leaky mode sets up. However, this dominant mode is not a bound mode with power flow dominating in the central line area with the amplitude monotonically decreasing laterally. In our case the central area, under the slot, has lower effective permittivity than has the outer area, between the parallel plates, where it equals $\varepsilon_{\mathrm{r}}$. Consequently the even dominant mode has only a real phase constant, which tends from zero to $\mathrm{k}_{\mathrm{TEM}}=\sqrt{ } \varepsilon_{\mathrm{r}} \mathrm{k}_{0}$, the propagation constant of the TEM parallel-plate mode, and can unattenuatedly propagate by itself. Its propagation constant computed in the full-wave approach holds even for frequency decreasing down to dc, as shown in Fig. 3, a zoomed part of Fig. 2 with removed normalization. The mode has the standing wave feature perpendicular to

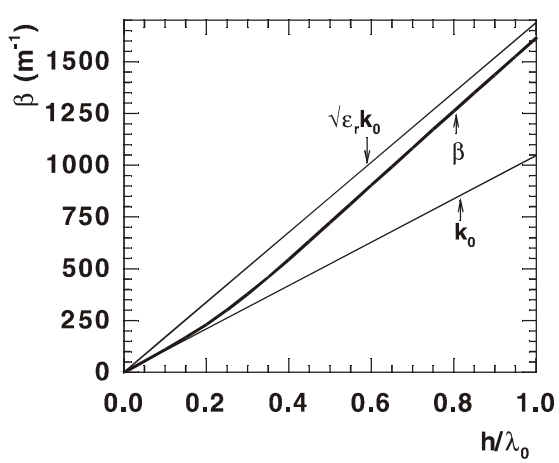

Fig. 3 Phase constant of the even dominant mode on the CBSL when $\mathrm{w}=6 \mathrm{~mm}, \mathrm{~h}=6 \mathrm{~mm}$ and $\varepsilon_{\mathrm{r}}=2.6$. 


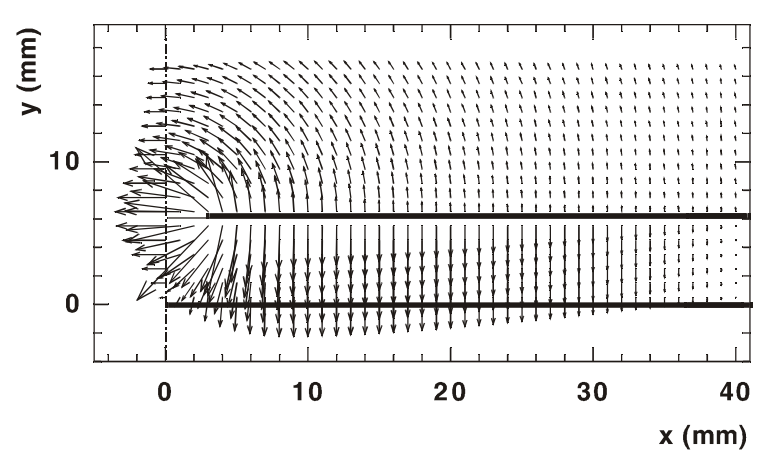

Fig. 4 Electric field of the even dominant mode in the cross-sectional plane when $\mathrm{w}=6 \mathrm{~mm}, \mathrm{~h}=6 \mathrm{~mm}$ and $\varepsilon_{\mathrm{r}}=2.6$ and $\mathrm{h} / \lambda_{0}=0.03$.

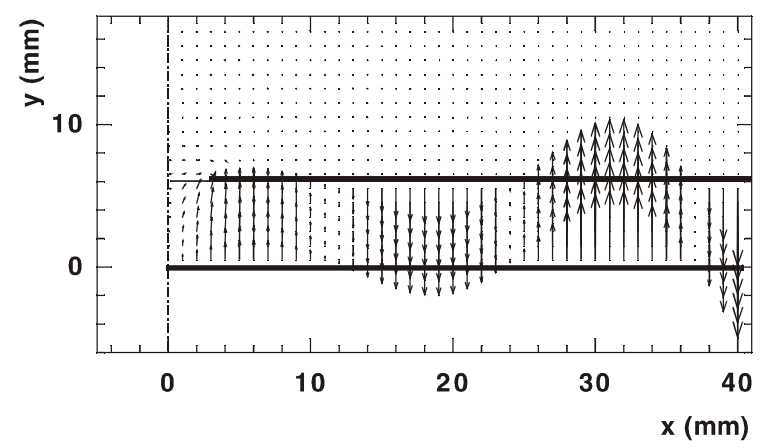

Fig. 5 Electric field of the 1 st even leaky mode in the cross-sectional plane when $\mathrm{w}=6 \mathrm{~mm}, \mathrm{~h}=6 \mathrm{~mm}$ and $\varepsilon_{\mathrm{r}}=2.6$ and $\mathrm{h} / \lambda_{0}=0.2$.
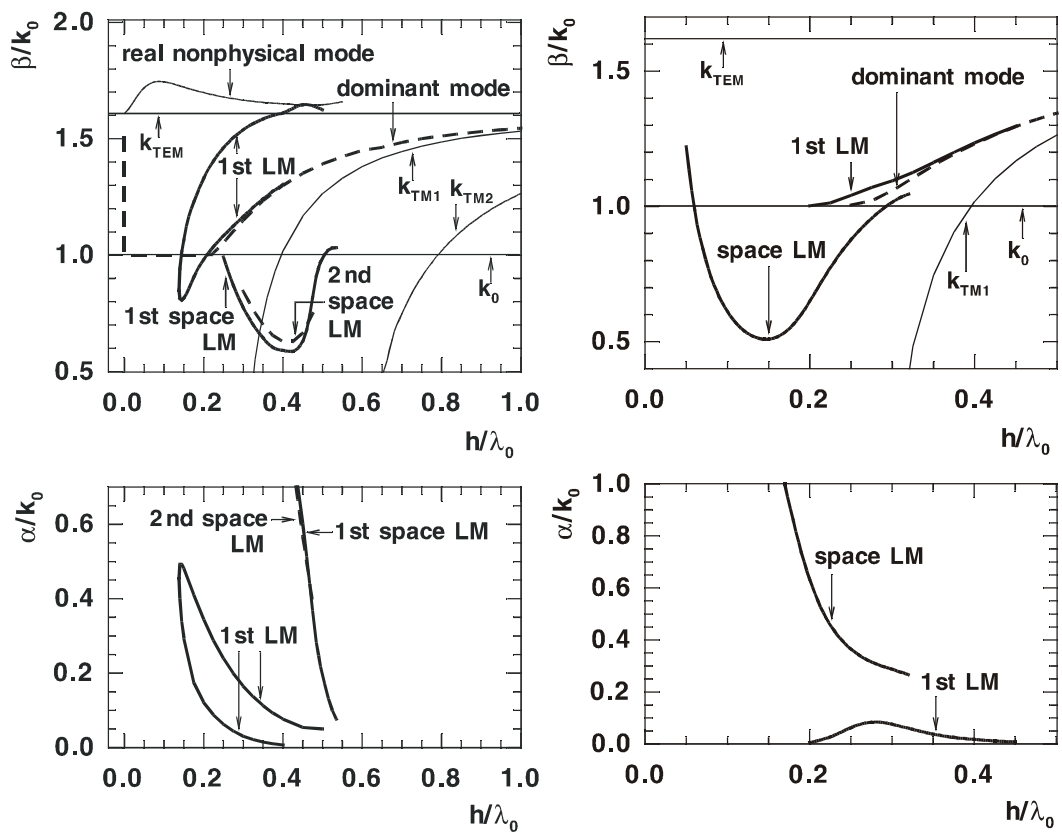

Fig. 6 Dispersion characteristics of the even modes on the CBSL with $\mathrm{w}=12 \mathrm{~mm}, \mathrm{~h}=6 \mathrm{~mm}$ and $\varepsilon_{\mathrm{r}}=2.6$. the line axis with laterally constant magnitude under assumption of a longitudinally and laterally unbounded, and lossless, CBSL. This conclusion follows from the solution of the dispersion equation of the sourceless line. Electric field of the even dominant mode is shown in Fig. 4.

Two branches of the real nonphysical solution of the dispersion equation which join at high $\beta / \mathrm{k}_{0}$ are also drawn in Fig. 2. The wider the slotwidth, the lower is this meeting point, see Fig. 6. The first leaky mode characteristic splits off from the real nonphysical solution. This mode becomes a physical wave at frequencies when its phase constant falls below $\mathrm{k}_{\mathrm{TEM}}$. Its electric field is shown in Fig. 5. Consequently we have a frequency band in which the only dominant mode propagates. A spurious nonphysical complex solution $\beta$ greater than $\mathrm{k}_{\mathrm{TEM}}$, splits off again from the real nonphysical solution, as plotted in Fig. 2. When the slotwidth grows, the split-off points of the true and spurious 1st leaky modes merge and become identical. This happens when the slotwidth of the CBSL studied here equals $7.001375 \mathrm{~mm}$. Far above this critical slotwidth, i. e., on a ,wide“ slotted CBSL, the spurious solution becomes physical and we have an ambiguous 1st leaky mode solution with two significantly different propagation constants, as shown in Fig. 6. Their field distributions are not shown here due to lack of space. This evolution of the dispersion characteristics was hitherto not known on other open planar transmission lines. The second leaky mode, carrying power as the TEM parallel-plate mode and simultaneously as the first $\mathrm{TM}_{1}$ mode, appears at higher frequencies. It converts to a physical leaky wave when its phase constant is greater than $\mathrm{k}_{0}$, and remains less than $\mathrm{k}_{\mathrm{TM} 1}$ as follows from Fig. 2. The even space leaky mode, the propagation constant of which is shown in Fig. 2, may also occur on this CBSL. Its relatively great leakage constant implies considerable leakage in the substrate, rather than high radiation efficiency. Two dispersion characteristics of the even space leaky modes are plotted in Fig. 6. The first one, which takes into account the simultaneous leakage in the TEM parallel-plate mode, is physical for $h / \lambda_{0}$ from 0.25 to

0.38 , but is inapplicable due to the great leakage constant. The second space leaky mode leaking in addition in the $\mathrm{TM}_{1}$ mode is physical for $\mathrm{h} / \lambda_{0}$ from 0.38 up to 0.51 . Indeed, the useful range of $\mathrm{h} / \lambda_{0}$, though with strong dispersion, is from 0.45 to 0.50 .

The appearance of the dispersion characteristics of the odd modes resembles those for the even modes. Widely-used feeding circuits cannot excite these odd modes on the „narrow“ slotted CBSL. Fig. 7, valid for $\mathrm{w} / \mathrm{h}=1.5$, shows a more interesting situation. The electric field distribution of the odd dominant mode is drawn in 
Fig. 8, while the field of the 1st odd leaky mode is shown in Fig. 9. At first glance they do not appear to differ much, since their phase constants are very close and the leakage constant is low. Consequently one wave masks the second wave, but the causes of their existence still differ. Space limitations prevent us from showing the even or odd space leaky mode electric field distribution.

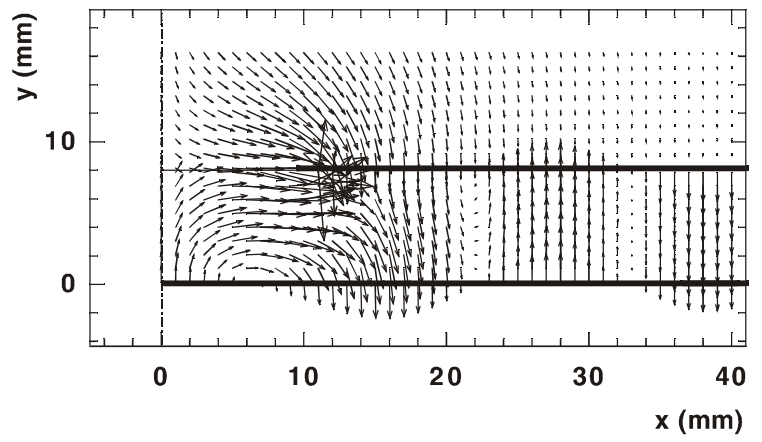

Fig. 8 Electric field of the odd dominant mode in the cross-sectional plane when $\mathrm{w}=20 \mathrm{~mm}, \mathrm{~h}=8 \mathrm{~mm}, \varepsilon_{\mathrm{r}}=2.6$ and $\mathrm{h} / \lambda_{0}=0.3$.

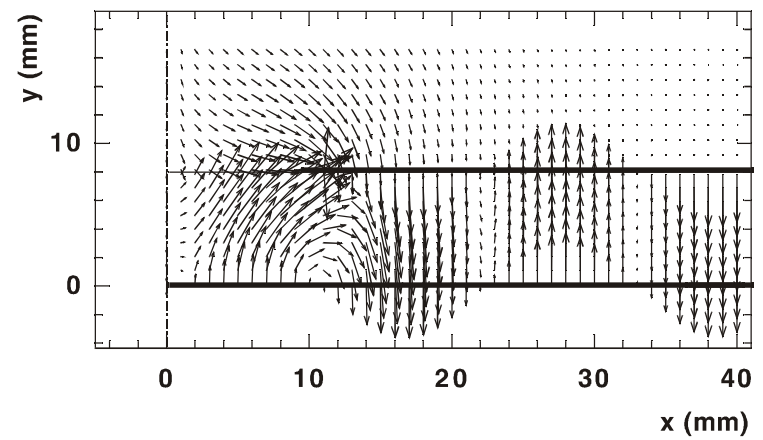

Fig. 9 Electric field of the 1st odd leaky mode in the cross-sectional plane when $\mathrm{w}=20 \mathrm{~mm}, \mathrm{~h}=8 \mathrm{~mm}, \varepsilon_{\mathrm{r}}=2.6$ and $\mathrm{h} / \lambda_{0}=0.3$.

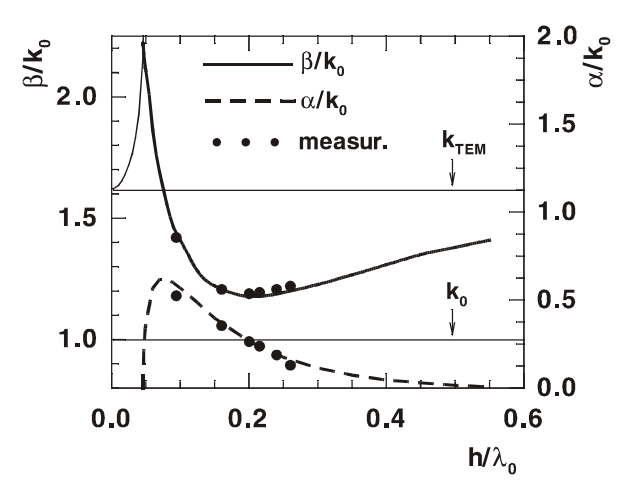

Fig. 10 Calculated and measured phase and leaky constants of the 1st leaky wave on the CBSL with $\mathrm{w}=6 \mathrm{~mm}, \mathrm{~h}=6 \mathrm{~mm}$, and $\varepsilon_{\mathrm{r}}=2.6$. these two waves was measured at the edge of the substrate. The calculated and measured halfwavelengths of the lateral standing wave were 31.0 and $28.9 \mathrm{~mm}$, respectively. The calculated and measured leakage angles of the 1st leaky wave were 42.2 and 42.5 degrees, respectively. Our measurement, in conjunction with the theoretically revealed even dominant wave, also explains the appearance of ripples in the record in Fig. 5 of [1].

\section{CONCLUSIONS}

It follows from the CBSL analysis that this line is not suitable for the purpose of transmission since the power flows in the area outside the central part of the line cross-section. This is the consequence of the effective permittivity placement in the cross-sectional plane. In addition the leakage in the substrate devalues the properties of the line. On the other hand the „wide“ slotted CBSL is a candidate for leaky wave antenna design, particularly operating with the first odd space leaky mode. The existence of even and odd dominant modes has been theoretically revealed and experimentally confirmed for the even mode. Ripples in the electric field record in [1] have been cleared up. An unexpected and previously unknown evolution of the dispersion characteristics dependent on the slotwidth has been revealed, including the double valued propagation constant of the even leaky mode. A careful analysis of the CBSL can still, particularly when covered on the top with a dielectric with greater permittivity, find new properties of the line with respect to size and permittivity modification.

\section{REFERENCES}

[1] H. Shigesawa, M. Tsuji, A. A. Oliner: Conductor-backed slot line and coplanar waveguide: Dangers and fullwave analysis. 1988 IEEE MTT-S Int. Microwave Symp. Dig., vol. 1, pp. 199-202, June 1988.

[2] H. Shigesawa, M. Tsuji, A. A. Oliner: Dominant mode power leakage from printed-circuit waveguides. Radio Science, vol. 26, no. 2, pp. 559-564, March-April 1991.

[3] N. K. Das, D. M. Pozar: Full-wave spectral-domain computation of material, radiation, and guided wave losses in infinite multilayered printed transmission lines. IEEE Trans. Microwave Theory and Tech., vol. MTT39, no.1, pp. 54-63, January 1991. 